\title{
StACKed Delta Design Of THREe-Phase PERMANENT-MAgnet FAult CURRENT Limiters
}

\author{
Mohamed Eladawy 1, 3*, and Ibrahim A. Metwally 2, 3 \\ ${ }^{1}$ Electrical Engineering Department, College of Engineering, Shaqra University, Riyadh, Saudi Arabia \\ ${ }^{2}$ Department of Electrical and Computer Engineering, College of Engineering, Sultan Qaboos University, \\ Muscat, Oman \\ ${ }^{3}$ Electrical Engineering Department, Faculty of Engineering, Mansoura University, Mansoura, Egypt
}

\begin{abstract}
This paper proposes an improvement for the dynamic performance of pre-saturated stacked permanent magnet biased three-phase fault current limiter (PMFCL) through COMSOL finite element simulation. The nonlinear demagnetization behaviour of the permanent magnet, especially in the upper part of the B-H curve with negative magnetic field intensity, has been modelled through the Jiles-Atherton method. This enables a realistic representation of the PMFCL dynamic behaviour throughout its entire operations of pre-fault, fault and fault removal, respectively. The experimental measurements were considered to validate the trends of the simulation outcomes during the entire operation of PMFCL. Extensive finite element simulation shows that the stacked design of PMFCL can increase the capability of fault current limiting with proper selection of the number and arrangement of the AC coils around the iron core (soft magnet). Results reveal that the division of AC coils into series differential connected sub coils, with an even number, can increase the limiting capability by increasing the AC coil number of turns, without exceeding the permissible tolerances of voltage drop and power losses. Moreover, this stacked design is subjected to parametric investigation for different fault types, either symmetrical or unsymmetrical, or even when changing the fault current peak value.
\end{abstract}

Keywords: COMSOL Multiphysics; iron core, neodymium; permanent magnet; pre-saturated core; fault current limiter.

\section{تصميم دلتا مكلس ثلاثى الأوجه لمحدات تيار الخطأ تعتمد على المقناطيسيات الائمة}

$$
\text { محمد العدوي* و إبر اهيم منولي }
$$

الملخص: يقترح هذا البحث تحسينًا للأداء الديناميكي لمحدد التيار الخطأ ثلاثي الأوجه سابق التشبع بواسطة المغناطيسيات

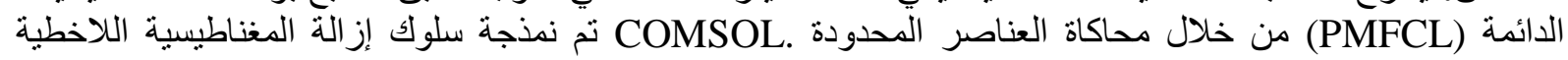

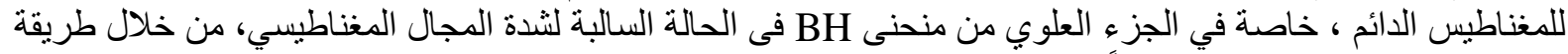

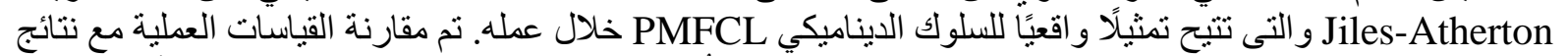

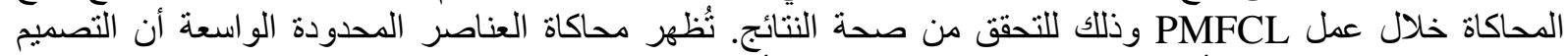
المكدس لـ PMFCL يمكن أن يزيد من عملية تحديد تيار الخطأ وذلك بالاختيار المناسب لعدد وترتيب ملفات التيار المتردد

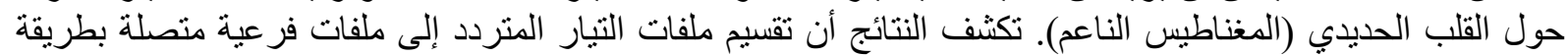

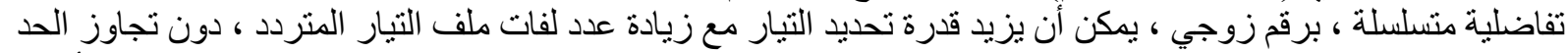

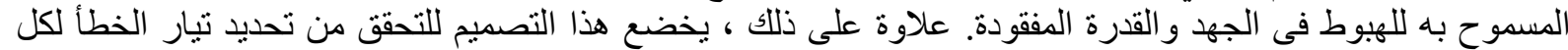

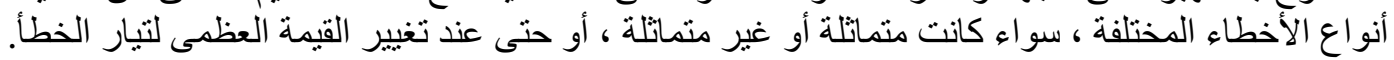

الكلماث المفتاحية: حزمة بر امج كومسول؛ القلب الحديدي؛ نيودنيوم؛ مغناطيس دائم؛ قلب سابق التشبع؛ محدد تيار الخطأ. 


\section{INTRODUCTION}

The fault current levels may be increased due to the progressive penetration of distributed generation into electrical power networks. Consequently, the power system reliability may be threatened due to these increased levels of fault current forthcoming the switchgear approaching limits. Therefore, upgrading infrastructure, circuit breakers, and/or survival switchgear represent a great challenge for utilities to conserve the reliability and full protection of power system against high levels of such fault current. This process of upgrading and/or bus splitting procedure can be considered a costly solution and bulky time consuming (Duggan 2006). Therefore, limiting fault current within the switchgear operating limits represents a leading candidate solution to avoid this upgrading process, which raises the importance of fault current limiter (FCL) technology (Eladawy et al. 2018; Moscrop 2013).

FCLs can be generally classified, according to their constructive technology, into solid-state FCLs (SSFCLs) (Radmanesh et al. 2016), quench-type superconducting FCLs (SFCLs) (Jia et al. 2016), and pre-saturated iron core FCLs (PCFCLs) (Eladawy et al. 2018; Moscrop 2013; Eladawy et al. 2019; Eladawy et al. 2020; Pannu et al. 2013; Yuan et al. 2017; Xiao et al. 2013, Zhao et al. 2007). SSFCLs utilize the power semiconductor switches for controlling the current flow through the circuit. An external controlling circuit, in general, is required for the detection of both fault and post-fault recovery events. However, the associated high power losses and unavoidable delays in fault detection and clearing process limit the reliability of this type of FCLs. In the quench-type SFCL, fault current limiting takes place due to the changeover from the initial state of very low impedance associated with superconductivity to very high impedance during fault occurrence. However, the SFCLs have operational difficulties, which limit their application, due to the required accompanying cryogenics, for preventing burnout of superconductor, and unavoidable delays in both fault reaction and post-fault recovery events (Radmanesh et al. 2016).

PCFCLs offer a self-governing performance of triggering for both fault occurrence and post-fault recovery events. The soft magnet (iron core), in such PCFCLs, is deeply saturated, which represents negligible impedance as the relative permeability $\mu_{r} \approx$ 1 during the healthy operating condition. This deeper saturation state can be offered by either a DC coil or permanent magnet (PM) (Eladawy et al. 2019, Eladawy et al. 2020). During fault condition, the highly counter-magnetic flux created by the fault current can in turn desaturate the soft magnet towards higher relative permeability values, which corresponds to a sharp increase of the soft magnet impedance and hence limiting the fault current. However, the DC coil can be either a simple nonsuperconducting one (i.e. copper coil) or a superconductor that permanently conserves its superconductivity state during the entire operation of the device. Consequently, this DC coil generates a highly magnetomotive force $(\mathrm{mm} f)$, which can be considered as the main drawback of such DC-biased PCFCLs due to the high power losses and high induced back electromotive force (emf) across the DC coil terminals during normal and fault conditions, respectively (Moscrop 2013).

Permanent magnet biased FCLs (PMFCLs) can replace and overcome the above-mentioned drawbacks of the traditional DC-biased PCFCL. In the three-phase PMFCL, the DC coils are replaced with $\mathrm{PM}$ for effective saturation of the associated soft magnet. Moreover, the simple construction of PM and its adequate behaviour for transition between healthy and faulty conditions encourage the spreading out of such PMFCLs, especially for increasing their operational capabilities with stacked designs. However, the truthful representation of PM with its nonlinear demagnetizing behaviour, especially in the upper part of its constructive material $B-H$ loop with negative magnetic field intensity, is a challenge for the design process of such PMFCLs (Eladawy et al. 2019). Consequently, finite element (FE) simulation provides an attractive tool, especially when validated with empirical results, for profoundly realizing the dynamic behaviour and tendencies of such PMFCLs.

This paper proposes a time-domain, threedimensional (3D) FE simulation of COMSOL Multiphysics package (COMSOL 2013) of a stacked design of three-phase PMFCL with performance investigation through, electric-circuit magnetic-field coupled model. However, the nonlinear demagnetizing representation of PM material, especially in the upper part of its $B-H$ hysteresis loop, is based on the well-known Jiles-Atherton (JA) modelling method. The aftermaths of JA model have been coupled with COMSOL to comprise the constitutive $B-H$ characteristics of PM exhibits nonlinear demagnetizing behaviour. Predominantly, the FE modelling technique has been verified and validated through comparison with experimental results of a given PMFCL. Furthermore, the proposed stacked design of PMFCL is devoted to the parametric investigation, especially the governing ones, to increase the operational capability in limiting fault current for distribution networks at different voltage levels. 


\section{DESIGN TOPOLOGY AND OPERATING PRINCIPLES}

\subsection{Design Topology}

Since the first conception of PCFCL, numerous system geometries have been inspected. Regardless of the different design topologies, the principle of operation remains the same. Fig. 1 shows the PMFCL included in the simplified test circuit (per phase), where the fault is represented by a switch (Eladawy et al. 2018). This switch is used to simulate a solid short circuit across the load terminal. Some of the common electrical parameters for the proposed delta-shaped PMFCLs are shown in Table 1 . The design arrangement of four AC coils and five NdFeB-N42 PM segments (4AC-5PM) three-phase delta-shaped PMFCL (Eladawy et al. 2020) is shown in Fig. 2(a). In this design, the AC coil of each phase has been divided into an even number of sub coils, conserving the total number of turns for each phase, as shown in Table 1 . These sub coils are equally distributed and wound on the periphery of soft magnet, which is represented by $1000 \mathrm{~mm}$ of side length equilateral triangle, forming the delta-shaped core, as shown in Fig. 2(a).

The subdivided coils of each phase are connected in series, with differential connection (Eladawy et al. 2020), to carry the line current of the protected power line. The uniformly distributed PM segments are sandwiched and arranged between the two soft magnet triangles throughout the delta-shaped design. The magnetization of each two adjacent PMs are in opposite directions, see parallel arrows in Fig. 2(a), in such a way to ensure forcing the soft magnet into a deeper magnetic saturation state. This leads to the soft magnet with very low impedance throughout the system normal operating condition. Additionally, this distribution of PMs in the magnetic circuit permits their force sources to bridge the distance between the soft magnets, hence resulting in a flux path with low reluctance. This supports effective saturation and PM protection during the healthy condition. Furthermore, this ensures effective fault current limiting in fault condition due to the high level of flux linkage crossing the magnetic circuit “demagnetization". Accordingly, the coils flux path is perpendicular to the PM magnetization direction, and along the soft magnet circumference track. Moreover, each two adjacent PM pieces have opposite magnetization directions, as shown in Fig. 2(a). This allows flux to return through the upper and lower triangles of the soft magnet. To prevent PM demagnetization, it is recommended to locate both the static $\mathrm{mmf}$ source and the AC mmf source in orthogonal positions, and include the PM into FCL magnetic circuit (Yuan et al. 2018).

The dimensions of PM and soft magnet are selected

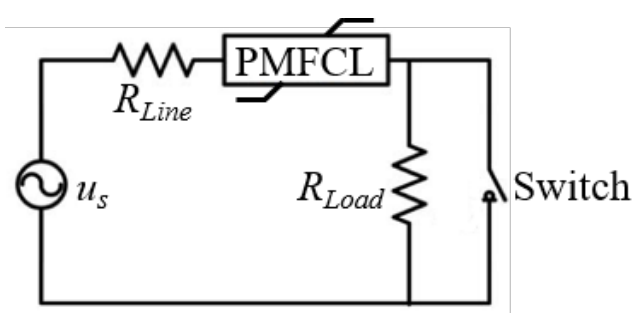

Figure 1. Simplified test circut (per phase) comprising the PMFCL with fau it representation switch.

Table 1. Common electrical parameters for delta shaped PMFCLs.

\begin{tabular}{cll}
\hline Symbol & Quantity & Value \\
\hline$u_{s}$ & $\begin{array}{l}\text { The rms value of the source line } \\
\text { voltage }\end{array}$ & $220 \sqrt{3} \mathrm{~V}$ \\
$R_{\text {Line }}$ & Line resistance & $0.1 \Omega$ \\
$R_{\text {Load }}$ & Load resistance & $3 \Omega$ \\
& Total number of AC coil turns of & 48 \\
$N_{a c}$ & each phase & \\
$a_{\text {coil }}$ & Copper wire cross-sectional area & $60 \mathrm{~mm}^{2}$ \\
$\sigma$ & Coil wire conductivity & $6 \times 10^{7}$ \\
$f$ & Power frequency & $50 \mathrm{~Hz}$ \\
\hline
\end{tabular}

such that the soft magnet is deeply saturated, which results in AC coils per phase with negligible inductance value during the healthy operating conditions. In addition, a substantial increase in the inductance per phase for the AC coil is obtained as a result of the significant rise of the soft magnet relative permeability, and hence its inductance, at fault occurs due to the demagnetization effect. However, the AC field induces eddy currents in the rare-earth neodymium PM (NdFeB-N42). Such an eddy current can be minimized by laminating the two equilateral triangles of the soft magnet core and shielding the electrically conductive PM. This will in turn minimize or prevent the PM demagnetization due to heat buildup.

The operational capability of PMFCL can be increased using stacked arrangements (one top of the other) of the soft magnet triangles. This stacked arrangement results in doubling the core thickness, as shown in Figs. 2(b) and 2(c). This will in turn increase the voltage drop value, due to the increase of AC coil inductance, during the healthy condition of operation. However, this may exceed the maximum permissible value of the voltage drop $(\approx 1 \%)$ stipulated by the utilities (Eladawy et al. 2020). Another multi-storey design can be obtained by aligning the magnetization direction in all storeys in the same direction, as shown in Fig. 2(d). In this design, the number of PM segments has been doubled and each head-to-head PM couples have the same direction of magnetization and opposite to the adjacent ones in opposite direction, as shown in Fig. 2(d). Each AC coil around the soft magnet is subdivided to eliminate the leakage 


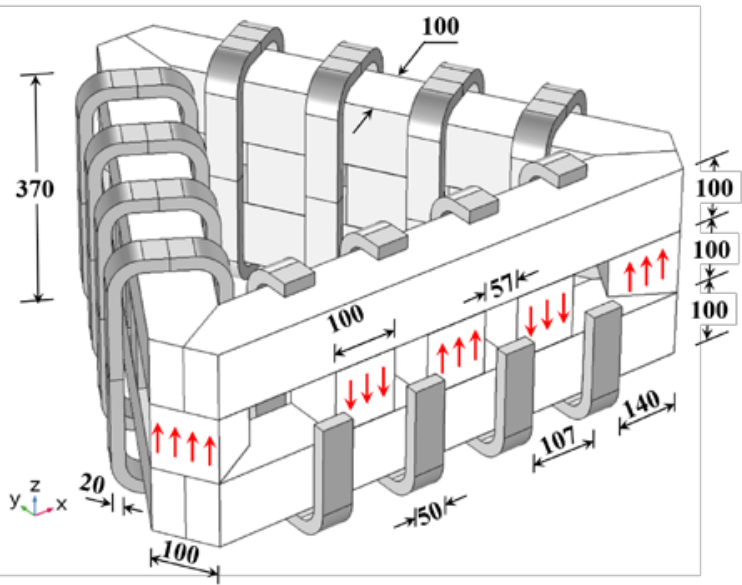

(a)

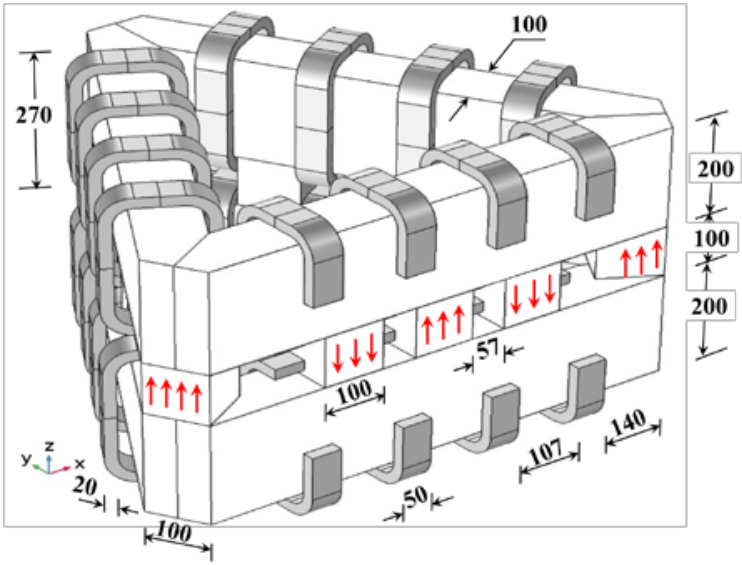

(c)

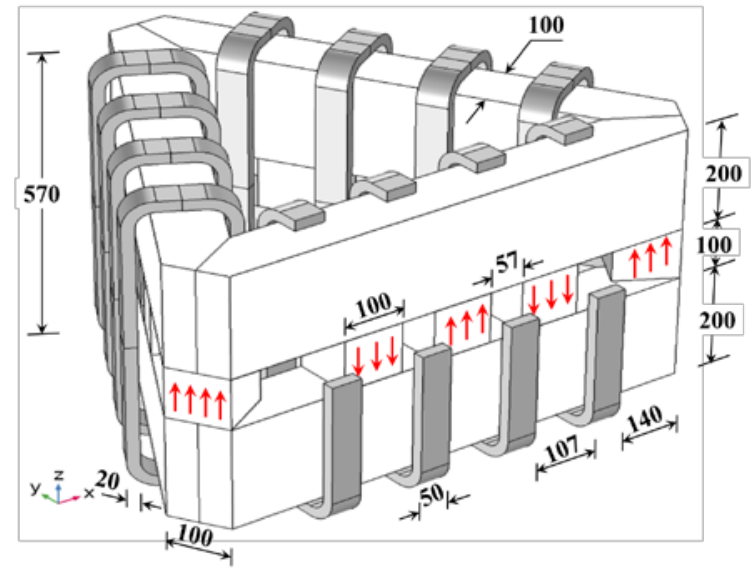

(b)

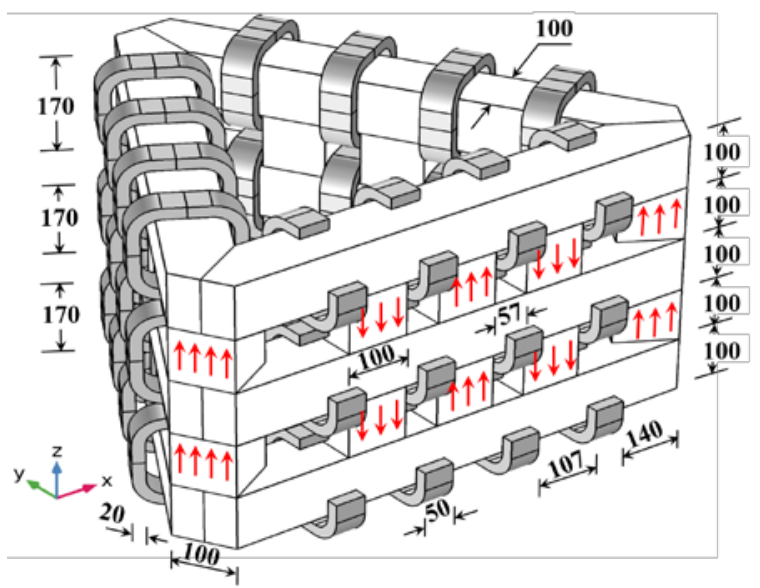

(d)

Figure 2. Three-dimensional view of three-phase PMFCLs with arrow directions of PMs magnetizations (a) 4AC-5PM reference delta-shaped design (Eladawy et al. 2020), (b) 4AC-5PM stacked delta, (c) 8AC-5PM stacked delta, and (d) 12AC-10PM multi-storey delta design. All dimensions are in $\mathrm{mm}$.

flux through the non-magnetic separation between the two soft magnets, forming the 12AC-10PM multistorey delta design. Therefore, the number of PMs, the height of the AC coil and its sub coils number strictly control the dynamic behaviour of the PMFCL.

\subsection{Operating Principles}

The operating principle of PMFCL remains the same as that of the DC-biased PCFCL. The rare-earth PM drives the soft magnet into deeper saturation with negligible inductance. Therefore, the PMFCL exhibits a non-limiting effect during the healthy condition of the system. Figure 2 illustrates that the PM is divided into smaller parts and distributed over the periphery of the soft magnet. This distribution provides effective saturation and reduces the heat build-up inside the $\mathrm{PM}$, hence avoiding the decrease of its magnetism at higher temperature (Coey 1996). In general, for each phase, two identical AC coils are required for effective limiting the two half cycles of the fault current. However, the even number of subdivided coils are connected in series, for each phase, with the differential connection. This differential connection decreases the voltage drop during the non-limiting condition of the PMFCL (Eladawy et al. 2018; Moscrop 2013; Eladawy et al. 2019, Eladawy et al. 2020).

The flux produced by the normal line current, during the normal operation, is sufficiently small to develop satisfactory demagnetizing $\mathrm{mmf}$, which is able to eliminate the strong magnetization of the soft magnet. Thus, the PMFCL approximately displays non-limiting behaviour. During fault occurrence, the highly generated $m m f$, by the fault current, can demagnetize the soft magnet towards higher values of $\mu_{r}$ in the $B-H$ curve linear region. A one-half cycle of fault current initiates half of the AC sub coils to come out of their initial strong saturation. Therefore, they are sharply rushed into a higher value of impedance. The subsequent fault current second half cycle drives the other part of the AC sub coils into a deeper saturation state, and vice versa, as shown in Fig. 3. At the fault removal instant, the PMFCL promptly recovers the initial state of strong saturation with negligible impedance and no limiting effect in less than 2 ms (Eladawy et al. 2020, Yuan et al. 2017). 


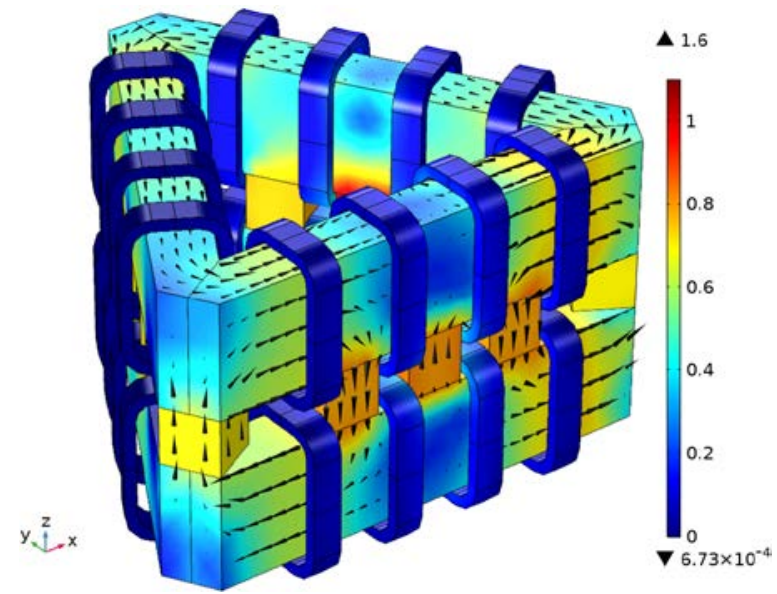

(a)

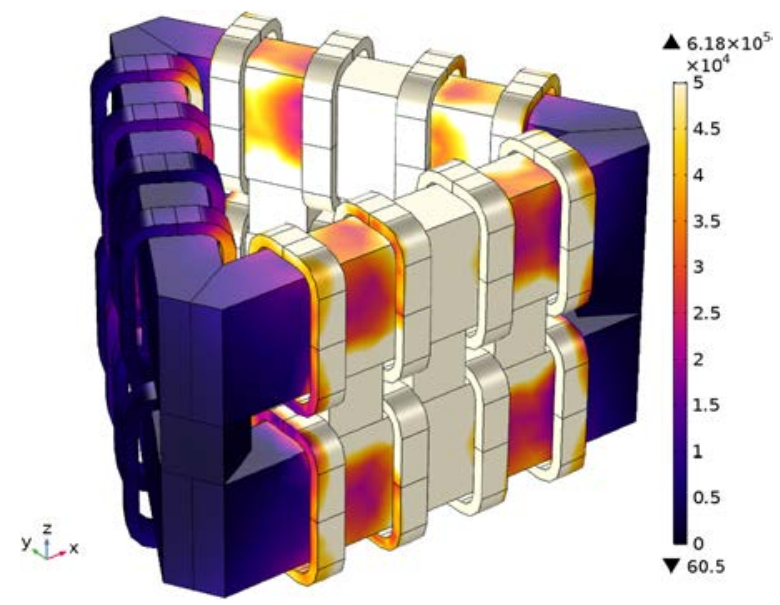

(b)

Figure 3. Surface plots at fault condition for (a) magnetic flux density $B$ in $(\mathrm{T})$ with cone directions of $B$, and (b) magnetic field intensity $H$ in $(\mathrm{A} / \mathrm{m})$, both at $t=0.09 \mathrm{~s}$, for the 8AC-5PM PMFCL stacked delta design. $N_{a c}=48$.

Nevertheless, the delta-shaped topology of the soft magnet provides magnetic symmetry configuration, and easier in manufacturing and assembly. This will, in turn, result in an approximately similar clipping ratio of fault current for any type of fault, and a similar voltage drop for the three phases in the healthy condition as the device is used in series without disturbing the balance condition of the power system. The nonlinear $B-H$ hysteresis loop is represented through JA method (Prigmore et al. 2013, Pop et al. 2011), and is considered as a base for further modelling of rare-earth neodymium PM (NdFeBN42) behaviour. More details about the characterizing $B-H$ hysteresis loop of NdFeB-N42 and the methodology of JA model can be found in (Eladawy et al. 2019; Eladawy et al. 2020), (Prigmore et al. 2013, Pop et al. 2011). JA method can effectively be used for modelling the anisotropic magnets exhibit remnant magnetic flux density. Accordingly, JA model is integrated with the FE simulation of COMSOL, in the time domain, for representing the nonlinear behaviour of PM, especially in the upper

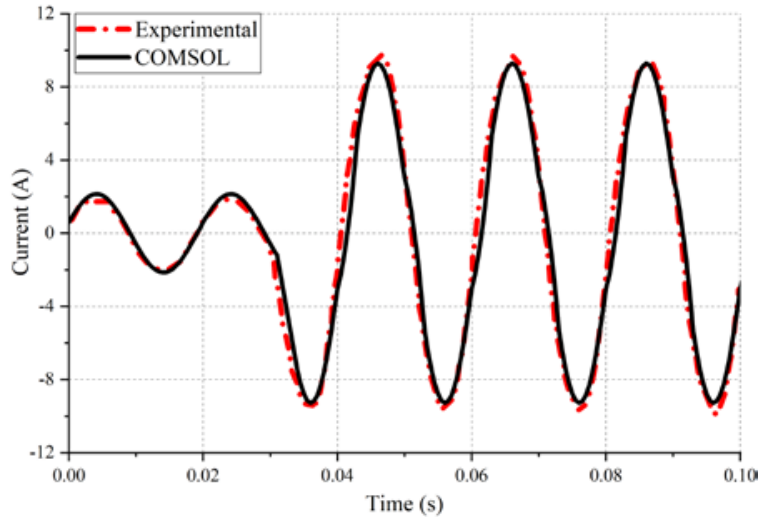

Figure 4. Comparison between the PMFCL experimental results of (Li et al. 2012) and the COMSOL FE simulation comprises the nonlinear behavior of PM.

part of its $B-H$ hysteresis loop with negative $H$ (Eladawy et al. 2019, Eladawy et al. 2020). Definitely, the constitutive $B-H$ relation represents the PM material using JA hysteresis model has been integrated into COMSOL (COMSOL 2013). Consequently, the foremost equations that have been modified to characterize a PM material exhibit a prescribed direction of remnant magnetic flux density. This enables updating the location of the intersecting point between both the characteristics of PM and soft magnet at each time step, and hence shows a realistic representation of the PMFCL behaviour during its entire operation.

Such coupled FE-JA model, which encompasses the nonlinear representation of PM through JA method, is predominantly validated with the experimental results of (Zou et al. 2008, Li et al. 2012), as shown in Fig. 4. However, the experimental setup in ( $\mathrm{Li}$ et al. 2012) has been modelled through the coupled FE-JA model, and the simulation results of this coupled model are compared with the experimental measurements as shown in Fig. 4. Figure 4 shows that the experimental results are in good agreement with simulation results with an error difference of $\pm 3 \%$ due to the missing AC coil dimensions ( $\mathrm{Li}$ et al. 2012). Therefore, the actual representation of PM demagnetizing behaviour through JA model in the upper part of the $B-H$ curve with negative magnetic field intensity offers truthful tendencies for the PMFCL dynamic behaviour in limiting fault current during normal and fault conditions.

\section{PERFORMANCE INVESTIGATION OF STACKED PMFCLS}

According to the above-mentioned operating principles of PMFCL, a 3D time-varying, COMSOL Multiphysics (COMSOL 2013) electric-circuit magnetic-field coupled FE model is reproduced. However, the three-phase stacked PMFCLs designs, Figs. 2(b), (c), and (d), are compared with the 
reference design of 4AC-5PM (Eladawy et al. 2020) shown in Fig. 2(a). However, the summarized parameters in Table 1 are common for all PMFCLs in Fig. 2. For the same total number of AC turns of each phase, the changes are through the thickness of the soft magnet, number of PM segments, and/or the number of AC sub coils. The line current reaches a peak value of $100 \mathrm{~A}$ and $3.1 \mathrm{kA}$, as shown in Fig. 2, (without any FCL), during the normal non-limiting and the fault conditions, respectively. The total simulation time is considered to be $0.14 \mathrm{~s}$, at which the fault has been started and removed at $t=0.04 \mathrm{~s}$ and at $t=0.1 \mathrm{~s}$, respectively.

In general, the clipping ratio $(k)$ of fault current, during the fault condition, is calculated as follows (Eladawy et al. 2019):

$k=\left(\hat{I}_{f}-\hat{I}_{L}\right) / \hat{I}_{f}$

where $\hat{I}_{f}$ and $\hat{I}_{L}$ are the prospective and limited peak values of fault current, respectively. This clipping ratio is termed to be calculated at the last peak of fault current, which represents the minimum value of the clipping ratio. Furthermore, the PMFCL dynamic performance is a strictly multi-parameter function in the physical and constructive parameters. It severely depends on the relative cross-sectional areas of the geometrical design topology of the soft magnet, the type of PM biasing, and the AC coil number of turns. Comprehensive FE simulations have been executed to identify the influence of such parameters on the dynamic performance of PMFCL (Eladawy et al. 2018; Moscrop 2013; Eladawy et al. 2019, Eladawy et al. 2020). Subsequently, the dynamic performance can be characterized by the clipping ratio $(k)$ of fault current, and the total power loss $\left(P_{\text {Loss }}\right)$ and the voltage drop across PMFCL terminals $\left(\Delta V_{F C L}\right)$ throughout the normal operation of the system.

\section{PERFORMANCE ANALYSIS 8AC-5PM STACKED DESIGN}

\subsection{Number of AC Coil Turns}

For increasing the current clipping ratio of the 8AC5PM design, the number of turns of AC coils per phase is increased $\left(N_{a c}=96\right)$, with conserving the other parameters in Table 1. Figures 5 (a), (b), and (c) show the three-phase currents of the 8AC-5PM stacked design, Fig. 2(c), during the entire simulation interval due to symmetrical three-phase, and unsymmetrical line-to-line and single line-to-ground faults, respectively. Figure 2(d) shows the voltage drop, across each phase of the AC coils, during the steady-state condition after fault removal, for the 8AC-5PM stacked delta PMFCL. It can be observed from Figs. 5 (a), (b), and (c) that the PMFCL promptly recuperates its initial saturation state after fault removal with a negligible impedance of the
PMFCL due to the strong saturation state offered by the distributed PM segments. However, the 8AC-5PM stacked delta PMFCL results in the minimum value of fault clipping ratio $k \approx 84.4 \%, k \approx 87.1 \%$ and $k \approx$ $81.8 \%$, for symmetrical three-phase, and unsymmetrical line-to-line and single line-to-ground faults, respectively. $\quad \Delta V_{F C L} \approx 0.89 \%$ and $P_{\text {Loss }} \approx$ $0.66 \%$ during the normal operating condition, without any observed significance on non-faulted phases (R) and ( $\mathrm{Y}$ and $\mathrm{B}$ ) in case of line-to-line and single lineto-ground fault, as shown from Fig. 6 (b) and (c), respectively. Irrespective of the duplication of the soft magnet volume, this design offers the enriched capability to limit fault current without exceeding both the power losses and voltage drop permissible tolerances.

Significant reduction in the voltage drop can be achieved by changing the arrangement of AC coils around the soft magnet with doubled soft magnet volume for the 8AC-5PM stacked design, as shown in Fig. 7, due to the effective saturation of the soft magnet and consequently reduces the current clipping ratio. The outcomes of FE simulations for the 4AC5PM PMFCL reference delta-shaped design (Eladawy et al. 2020), shown in Fig. 2(a) in accordance with Table 1, have been reproduced and the soft magnet volume has been taken as a reference for all other designs. This design will result in $k \approx 68 \%, \Delta V_{F C L} \approx$ $0.8 \%$ and $P_{\text {Loss }} \approx 0.39 \%$, as shown in Fig. 8. However, any further increase in soft magnet volume, as shown in Fig. 2(b), or $N_{a c}$ will in turn increase the steadystate voltage drop and power losses. This can be referred to the increase in the AC coil impedance due to the increase in the coil area or its number or turns. Therefore, the 4AC-5PM stacked delta design exhibits some limiting effect throughout the normal operating condition. Conserving the same number of AC turns, the multi-storey delta design of 12AC-10PM gives the lowest fault current clipping ratio, voltage drop, and power losses, as shown in Fig. 8. This referred to the deeper saturation state of the soft magnet due to the increase of PM segments. These lower values of $\Delta V_{F C L}$ encourages increasing the $\mathrm{AC}$ coil turns for increasing the clipping ratio of fault current without exceeding the permissible limits of voltage drop and power losses. Figure 8 shows that the 8AC-5PM stacked delta design offers adequate characteristics in limiting fault current while conserving the same number of AC coil turns for each phase, the number of PM segments and increasing the volume of the soft magnet.

Figure 7 shows the dynamic performance of 8AC5PM PMFCL with changing the AC coil number of turns per phase. However, increasing $N_{a c}$ leads to an increase in the AC coil inductance. Consequently, the PMFCL offers a higher clipping ratio of fault current with increasing $N_{a c}$ due to the increase of fault 
impedance, as shown in Fig. 8. Accordingly, this increase of Nac leads to an increase in both the voltage drop and power losses throughout the normal operation of the system, due to the increase of steadystate impedance (Eladawy et al. 2018, Moscrop 2013). Therefore, the AC coil number of turns can be considered as a crucial parameter, which controls the PMFCL entire operating conditions.

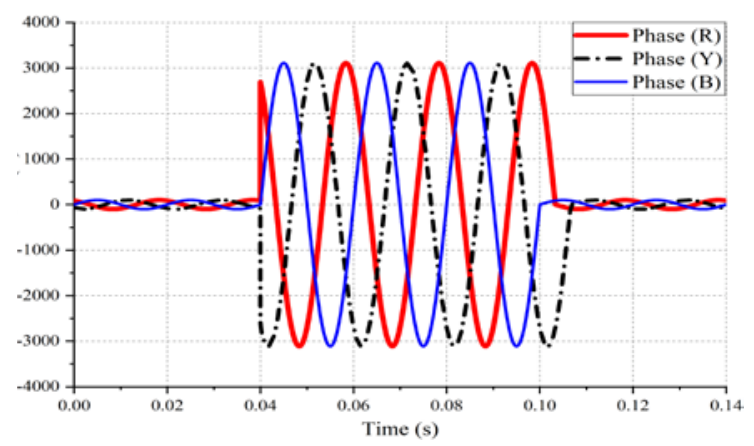

Figure 5. Three-phase currents of the test circuit without any PMFCL.

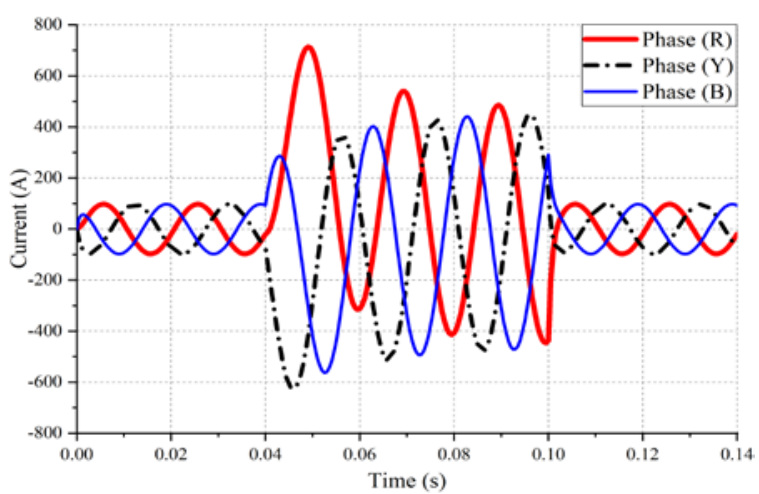

(a)

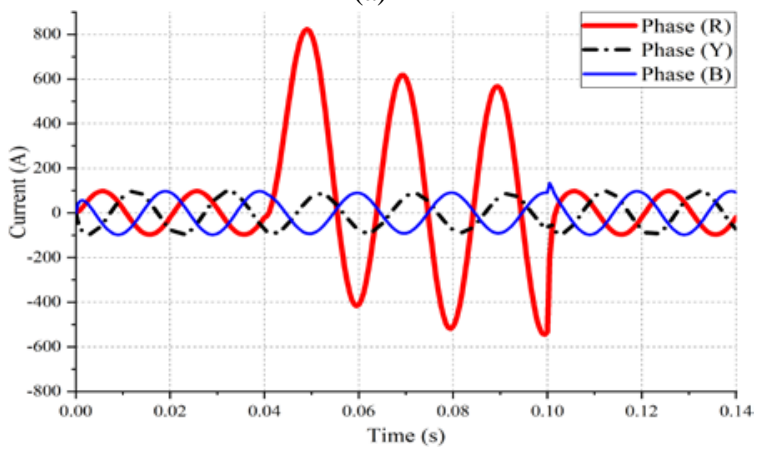

(c)

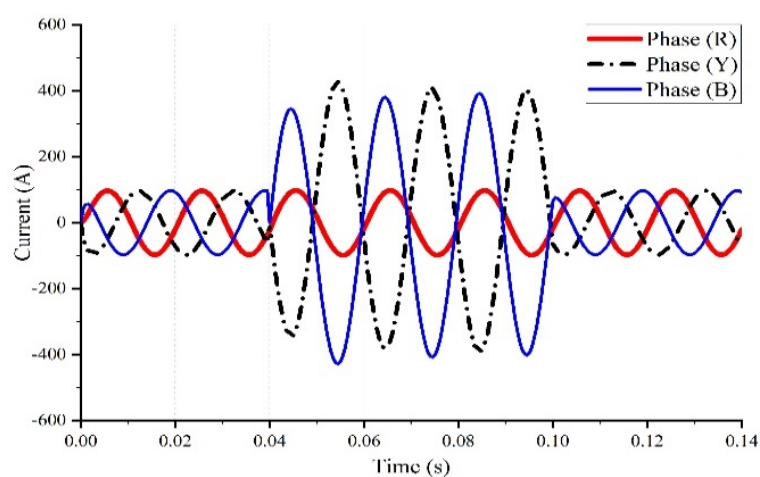

(b)

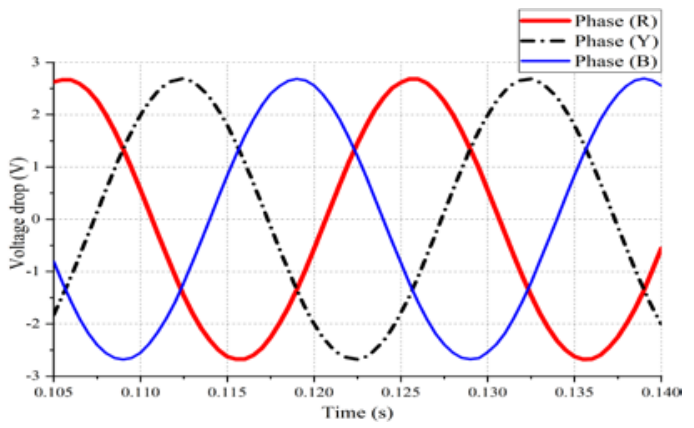

(d)

Figure 6. Clipping effect of fault current and voltage drop waveforms for the 8AC-5PM stacked delta PMFCL due to symmetrical three-phase fault, line-to-line fault and single-line-to-ground fault. $N_{a c}=96$. (a) Three-phase fault currents, (b) Line-to-line fault, (c) Single-line-to ground fault currents, and (d) Voltage drop.

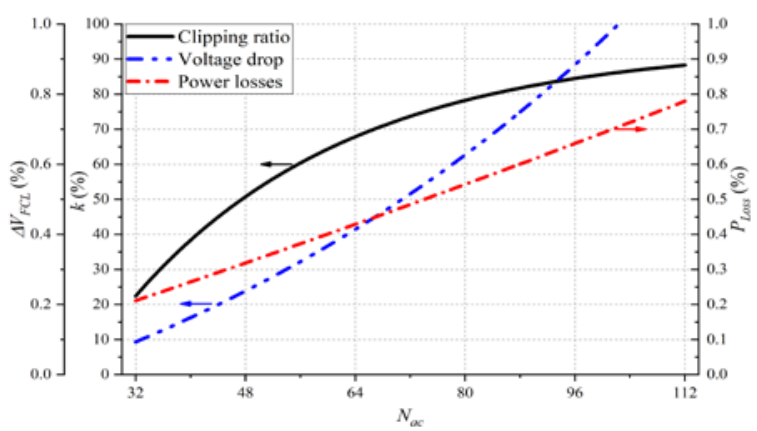

Figure 7. Variation of the dynamic performance of 8AC5PM PMFCL stacked design with changing the AC coil number of turns.

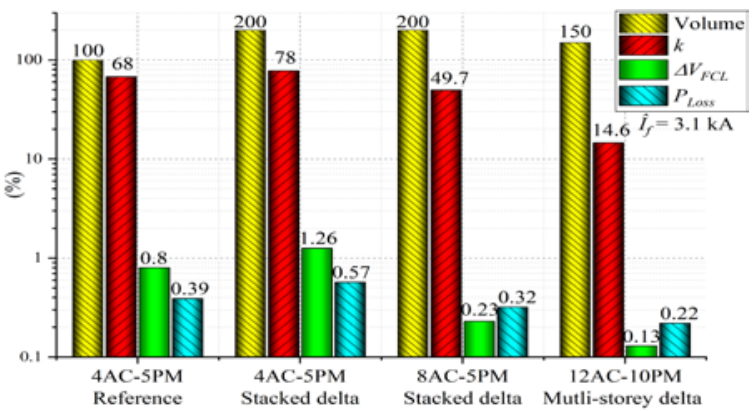

Figure 8. Comparison between the different proposed stacked designs of PMFCLs. Volume is referred to the reference design of 4AC-5PM delta design (Eladawy et al. 2020). $N_{a c}=48$. 


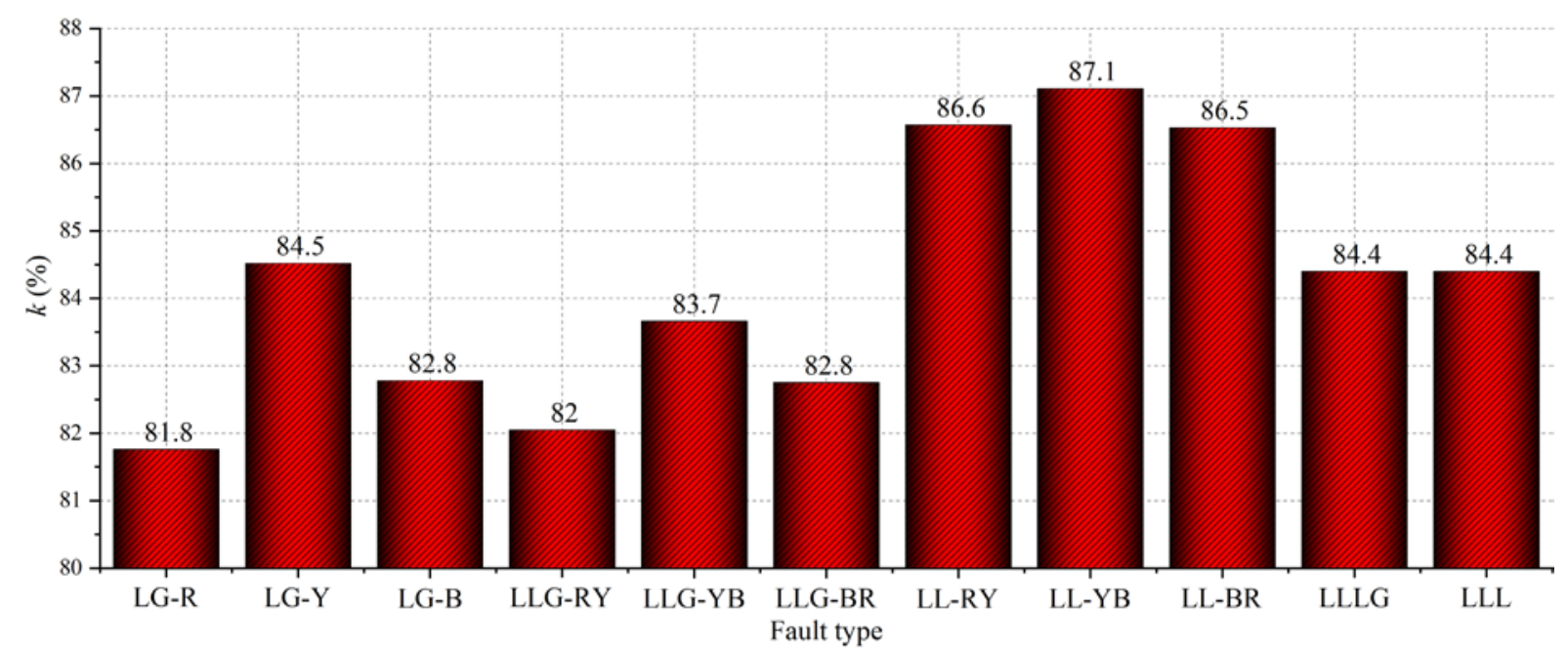

Figure 9. Fault current clipping ratio of three-phase 8AC-5PM PMFCL for different fault types. $N_{a c}=96$.

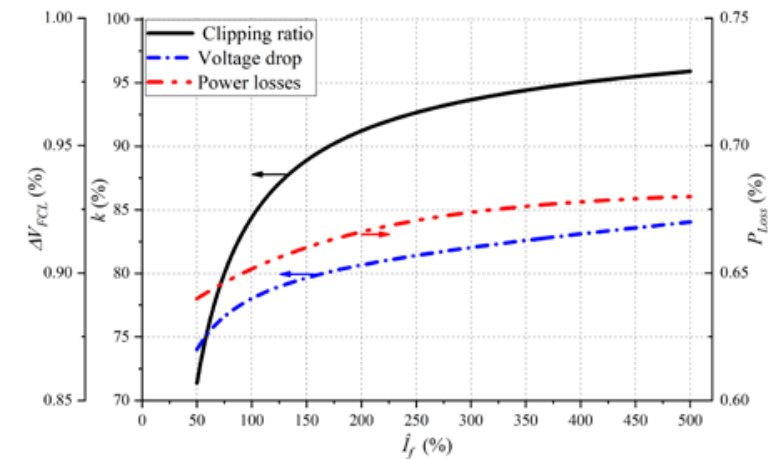

Figure 10. Variation of the dynamic performance of 8AC5PM PMFCL stacked design with changing the fault current peak value. $N_{a c}=96$.

\subsection{Fault Type and Fault Current Peak Value}

For ensuring the reliability of the three-phase 8AC5PM PMFCL, different unsymmetrical faults have been tested through extensive FE simulations. These unsymmetrical faults that comprise line-to-ground (LG), double line-to-ground (LLG), and line-to-line (LL) are compared with those for the symmetrical three line-to-ground (LLLG) and three-line (LLL) faults. Figure 9 shows the satisfactory capability of the 8AC-5PM PMFCL in limiting the fault current $(k$ $\geq 81.8 \%$ ) for different fault types, when $N_{a c}=96$, without exceeding the permissible tolerances of both the voltage drop and power losses. The small difference in the current clipping ratio for different phases (for the same fault type) can be referred to as the point on wave switching. Moreover, extensive FE simulations show that the healthy phases are tremendously unaffected by the faulted phase in the case of unsymmetrical faults.

Consequently, decreasing the line resistance $\left(R_{\text {Line }}\right)$ will in turn increase the fault current peak value.
Referring to Table 1 parameters and for $N_{a c}=96$, the fault current has been changed up to $500 \%$ of its reference value $\left(\hat{I}_{f}=3.1 \mathrm{kA}\right)$, as shown in Fig. 10 . It can be observed from Fig. 10 that the increase of fault current will in turn lead to higher demagnetization of the soft magnet, which leads the operating point towards the higher relative permeability in the $B-H$ curve linear zone. Thus, this leads to higher fault impedance and higher fault current clipping ratio as shown in Fig. 10. Furthermore, decreasing the line resistance leads to an increase in the steady-state line current, which will in turn slightly increase the voltage drop and the power losses. However, neither the voltage drop nor the power losses exceed their permissible tolerances due to the effective saturation of the soft magnet throughout the healthy condition of the system. Subsequently, the 8AC-5PM stacked design of PMFCL offers improved capability in limiting any fault type even when the fault current peak value is increased.

Accordingly, the connection between the AC sub coils is a crucial design parameter that significantly affects the dynamic performance of the PMFCL. However, the cumulative connections of sub coils increase the overall AC coil inductance during the non-limiting operation. This leads to an increase in the voltage drop across the PMFCL terminals. Moreover, the AC coil wire's cross-sectional area significantly affects the power losses, especially during the non-limiting condition. Therefore, it should be regulated to obtain power losses percentage with the specified tolerance by the utility. However, similar tendencies with different values are obtained with the 12AC-10PM PMFCL. This ensures that the rearrangement of the AC coils around the soft magnet can be very useful in extending the range of operation of the stacked or multi-storey PMFCL designs. 


\section{CONCLUSION}

The capability of fault current limiting of the threephase delta-shaped PMFCL design is enhanced by introducing the stacked and multi-storey delta-shaped designs. Extensive 3D, time domain, electric-circuit magnetic-field coupled model, FE simulations of COMSOL Multiphysics have been carried out taking into consideration the realistic representation of the PM demagnetization behaviour in the upper part of the $B-H$ hysteresis loop with negative magnetic field intensity. NdFeB-N42 PM is only considered to bias the soft magnet. The PM demagnetizing behaviour is represented through the $B-H$ loop of JA model. The PM segments are sandwiched with a uniform distribution between two equilateral triangles. For effective saturation, the magnetization directions are kept opposite for each two adjacent PMs. The AC sub coils of each phase are placed at the air gaps between each two adjacent PMs, and wound around the soft magnet with a differential series connection.

Increasing the soft magnet cross-sectional area leads to an increase in both the clipping ratio of fault current and the voltage drop across the terminals of AC coils. This limits the capability of the PMFCL in limiting the fault current to be within the specified tolerance of the voltage drop. The division of the AC coils, into an even number, of sub coils and locating them at the air gaps between the soft magnets leads to the reduction of the inductance of such coils during the non-limiting performance of the PMFCL. This has the effect of reducing the voltage drop. Therefore, this gives the opportunity to increase the AC coils number of turns. This will in turn increase the capability of fault current limiting using the stacked or multi-storey configurations. The three-phase 8AC-5PM stacked delta design shows adequate dynamic performance in limiting the fault current without duplicating the PM segments number. Results reveal that this design has enriched the capability to limit all fault types, either symmetrical or unsymmetrical, with increased peaks of fault currents.

\section{CONFLICT OF INTEREST}

The authors declare no conflict of interest.

\section{FUNDING}

No funding was received for this study.

\section{REFERENCES}

Coey JMD (1996), Rare-earth Iron Permanent Magnets, Clarendon Press, ISBN: 9780198517924.

COMSOL Group Ltd., Stockholm, Sweden (2013), COMSOL Multiphysics software package V.4.4.
Duggan PM (2006), Integration issues for fault current limiters and other new technologies-A utility perspective. in Proc. IEEE Power Eng. Soc. Gen. Meeting, Montreal, QC, Canada: 1-3.

Eladawy M, Metwally IA (2018), Design and Performance Analysis of Single-Phase Presaturated Core Fault Current Limiters, IEEE Trans. Power Del., 33(6): 2603-2612.

Eladawy M, Metwally IA (2018), A Novel Five-Leg Design for Performance Improvement of ThreePhase Presaturated Core Fault Current Limiter, IEEE Trans. Magn., 54(7), Sequence No. 8001410: 1-10.

Eladawy M, Metwally IA (2020), Compact Designs of Permanent-Magnet Biased Fault Current Limiters, IET Electr. Power Appl., (3): 471 - 479.

Eladawy M, Metwally IA (2018), Design and Performance Analysis of Single-Phase Presaturated Core Fault Current Limiters, IEEE Trans. Power Del., 33(6): 2603-2612.

Eladawy M, Tarhuni NJ, Metwally IA (2019), Transient Dynamic Analyses of Presaturated Core Fault Current Limiters through Flux and Inductance versus Current Modelling, IET Electr. Power Appl., 13 (9): 1263-1272.

Jia Y, Yuan J, Shi Z, Zhu H, Geng Y, Zou J (2016), Simulation method for current-limiting effect of saturated-core superconducting fault current limiter, IEEE Trans. Appl. Supercond., 26(4), Art. no. 5601404.

Li Q, Xu J, Zou L, et al. (2012), Modelling methodology and experimental verification of the permanent-magnet-biased saturation-based fault current limiter, IET Electr. Power Appl., 6(8): 504-512.

Moscrop JW (2013), Experimental Analysis of the Magnetic Flux Characteristics of Saturated Core Fault Current Limiters, IEEE Trans. Magn., 49(2): 874-882.

Pannu M, Valent Y, Garbi U (2013), Pre-saturated Core Fault Current Limiter, Australasian Universities Power Engineering Conference, AUPEC, Hobart, TAS, Australia, 29 Sept. - 3 Oct.

Pop NC, Caltun OF (2011), Jiles-Atherton magnetic hysteresis parameters identification, Acta Phys. Polonica A, 120(3): 491-496.

Prigmore J, Mendoza JA, Karady GG (2013), Comparison of Four Different Types of Ferromagnetic Materials for Fault Current Limiter Applications, IEEE Trans. Power Del., 28(3): 1491-1498.

Radmanesh H, Fathi SH, Gharehpetian GB, Heidary A (2016), Bridge-type solid-state fault current limiter based on AC/DC reactor, IEEE Trans. Power Del., 31(1): 200-209.

Xiao H et al. (2011), Analysis of transient overvoltage in $220 \mathrm{kV}$ saturated core HTS FCL, IEEE Trans. Magn., 47(10): 2620-2623.

Yuan J, et al. (2017), A novel hybrid saturated core fault current limiter topology considering permanent magnet stability and performance,” 
IEEE Trans. Magn., 53(6), Art. no. 8400304.

Yuan J, Zhou H, Gan P, et al. (2018), A novel concept of fault current limiter based on saturable core in high voltage DC transmission system, AIP Advances, 8(5), paper 056636: 1-6.

Zhao C, Wang S, Qiu J, et al. (2007), Transient Simulation and Analysis for Saturated Core High
Temperature Superconducting Fault Current Limiter, IEEE Trans. Magn., 44(4): 1813-1816.

Zou L, Liu H, Li Q, Siew WH (2008), Analysis and Simulation of the PMFCL Based on Coupled Field-circuit Modeling Methodology, in Proc. Int. Conf. High Voltage Eng. Appl., Chongqing, China, 9-13: 498-502 